\title{
Conductive Cotton Prepared by Polyaniline In Situ Polymerization Using Laccase
}

\author{
Ya Zhang • Aixue Dong • Qiang Wang • Xuerong Fan • Artur Cavaco-Paulo • \\ Ying Zhang
}

Received: 19 March 2014 / Accepted: 22 July 2014 /

Published online: 7 August 2014

(C) Springer Science+Business Media New York 2014

\begin{abstract}
The high-redox-potential catalyst laccase, isolated from Aspergillus, was first used as a biocatalyst in the oxidative polymerization of water-soluble conductive polyaniline, and then conductive cotton was prepared by in situ polymerization under the same conditions. The polymerization of aniline was performed in a water dispersion of sodium dodecylbenzenesulfonate (SDBS) micellar solution with atmospheric oxygen serving as the oxidizing agent. This method is ecologically clean and permits a greater degree of control over the kinetics of the reaction. The conditions for polyaniline synthesis were optimized. Characterizations of the conducting polyaniline and cotton were carried out using Fourier transform infrared spectroscopy, UV-vis spectroscopy, cyclic voltammetry, the fabric induction electrostatic tester, and the far-field EMC shielding effectiveness test fixture.
\end{abstract}

Keywords Functional finish $\cdot$ Conductive cotton · Aspergillus $\cdot$ Laccase-catalyzed synthesis $\cdot$ In situ polymerization

\section{Introduction}

Polyaniline (PANI) is one of the most important members in the class of intrinsically conducting polymers due to its favorable electrical properties and high thermal and chemical stability [1]. It is used in many applications in various fields including antistatic coatings [2], anticorrosion protection [3], organic lightweight batteries [4], and biosensors [5].

Polyaniline is composed of a reduction unit and an oxidation unit. The combination of benzenoid and quinoid rings leads to three typical oxidation states: a reduction state $(x=1)$, an

Y. Zhang $\cdot$ A. Dong $\cdot$ Q. Wang $(\bowtie) \cdot$ X. Fan $\cdot$ Y. Zhang

Key Laboratory of Science and Technology of Eco-Textile, Ministry of Education, Jiangnan University, Wuxi 214122 Jiangsu, China e-mail: qiang_wang@163.com

Q. Wang · A. Cavaco-Paulo International Joint Research Laboratory for Textile and Fibre Bioprocesses, Jiangnan University, Wuxi 214122 Jiangsu, China

A. Cavaco-Paulo

Department of Biological Engineering, University of Minho, Campus de Gualtar, 4710-057 Braga, Portugal 
oxidation state $(x=0)$, and a half oxidation state $(x=0.5)$, which make the conjugation mechanism of PANI different from that of other conducting polymers [6]. The half oxidation state of polyaniline is the only one that can be conductive after doping. The structure of polyaniline is given as follows ( $x$ depends on the oxidation degree of the polymer chain and $n$ is the repeating unit):

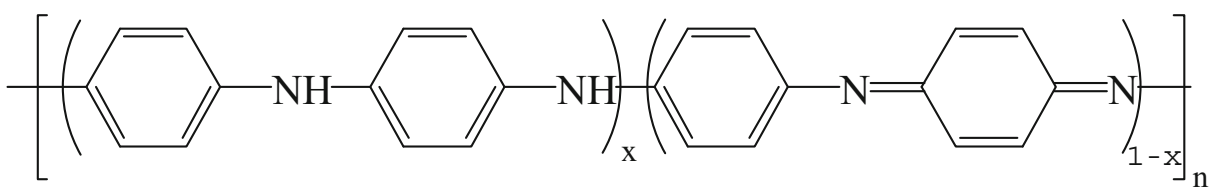

Electroconductive polyaniline is commonly produced by oxidizing an aniline monomer in strongly acidic media using ammonium persulfate as an initiator of oxidative polymerization [7]. On the basis of this traditional chemical synthesis method, conductive polyaniline nanofibers are commonly prepared by electrospinning, which includes ultrafiber electrospun from pure polyaniline solutions and their mixtures with various polymers $[8,9]$. However, the reaction is not kinetically controllable and the polyaniline obtained is not processable because of the poor solubility of polyaniline in conventional organic solvents. In addition, the reaction is harmful to the environment as a result of further steps of final polymer purification and high concentrations of acid and ammonium persulfate.

By contrast, enzymatic catalysis, which does not have an induction period as does chemical initiation [10], is more attractive as an alternative method for the synthesis of conductive PANI because it is performed under milder conditions and is kinetically controllable [11]. Laccases are multicopper oxidoreductases, which can catalyze the oxidation of a wide range of substrates with the concomitant reduction of oxygen to water [12]. In contrast to chemical and peroxidase-based oxidative polymerization, laccase-catalyzed reactions not only are ecologically clean but also avoid the use of $\mathrm{H}_{2} \mathrm{O}_{2}$, as atmospheric oxygen serves as the oxidizing agent [13]. Moreover, the peroxidases will be inactivated at high concentrations of $\mathrm{H}_{2} \mathrm{O}_{2}$ [14]. Polymerization of conductive polyaniline by a laccase-catalyzed method in a water dispersion of sodium dodecylbenzenesulfonate micellar solution was first reported by Streltsov et al. in 2007 [11]. In their work, the micelles of sodium dodecylbenzenesulfonate (SDBS) served as linear templates and dopants and facilitated the head-to-tail coupling of the monomers, thus causing an increase in the conductivity of the polyaniline. Meanwhile, the long alkyl SDBS chains enhanced the solubility of the polymer in common solvents.

In the field of electronics, smaller and more lightweight items are always ideal. The benefits of natural textile materials may be utilized to create devices that have the requisite conductive properties if conductive PANI could be immobilized on the surfaces of textiles $[15,16]$. There are simple electronic devices that could benefit by using the lightweight flexibility that some textile materials provide. Research such as this has potential health and monitoring applications such as a uniform with cloth sensors embedded in the actual material that track heart rate, body temperature, movement, and more in real time.

Conductive nanocoatings are usually applied to inorganic materials such as silicon [9]. For this paper, laccase-catalyzed polymerization of aniline was performed in a water dispersion of SDBS. Conductive cotton was prepared by in situ polymerization under the same reaction conditions. This new system gives researchers a better understanding of how to apply conductive PANI to textiles in order to turn them into conductive devices. The physicochemical properties of the PANI/SDBS complex and the antistatic properties of conductive cotton were also studied. 


\section{Materials and Methods}

Materials

Laccase Denilite from Aspergillus (EC1.10.3.2) was kindly supplied by Novozymes (Shanghai, China). The activity of laccase had been measured spectrophotometrically and was described in a previous work [17]. One unit of laccase was defined as the amount of enzyme required to oxidize $1 \mu \mathrm{mol}$ of ABTS per minute at room temperature. Nafion, a perfluorosulfonate linear polymer, exploited by DuPont Company, was purchased from Sigma-Aldrich. Aniline, SDBS, and all other reagents used were of analytical grade from Sinopharm Chemical Reagent Co., Ltd. (Shanghai, China), without further purification. All solutions were prepared with deionized water.

\section{Laccase-Catalyzed Synthesis of Conducting Polyaniline}

A 0.35 -mg SDBS sample was dissolved into $100 \mathrm{~mL}$ of $50 \mathrm{mM} \mathrm{Na}_{2} \mathrm{HPO}_{4}$-citric acid buffered solution ( $\mathrm{pH} 4.5$ ), followed by the addition of aniline with constant stirring. The stirring was stopped when the aniline was completely dissolved. The reaction was initiated by the addition of $50 \mu \mathrm{L}$ of laccase solution. The reaction vessels were put into a freezer at $5{ }^{\circ} \mathrm{C}$ for $24 \mathrm{~h}$ to complete the polymerization process, and UV-vis spectra of the reaction products were recorded at regular intervals.

The same amount of acetone $(100 \mathrm{~mL})$ was added to the PANI/SDBS dispersion to collapse the micelles and precipitate the PANI by adjusting the hydrophilic-hydrophobic balance of the system. The precipitate was collected by centrifugation and washed twice with $100 \mathrm{~mL}$ of acetone and $100 \mathrm{~mL}$ of $50 \%(\mathrm{v} / \mathrm{v})$ acetone/water mixture each time to remove the unreacted chemicals and aniline oligomers. The final purified polymer complex was dried in an oven at $60{ }^{\circ} \mathrm{C}$ for $24 \mathrm{~h}$ for further characterization.

\section{Conducting Cotton Prepared with In Situ Polymerization}

A sample of cotton was put into the reaction solution before the addition of the laccase. Stirring was stopped when the solution was homogeneous and stable. The reaction was initiated by the addition of the laccase. Other reaction conditions are in accordance with the conditions mentioned above.

The cotton sample was taken out after the micelles were collapsed. The cotton was repeatedly washed with acetone and deionized water before being dried in an oven at $60{ }^{\circ} \mathrm{C}$. The same reaction without laccase was used as a reference.

\section{UV-Vis Spectra Analysis}

UV-vis spectra of the PANI complexes were recorded using a UV-2808S UV-vis spectrophotometer (Unicosh, China) in the range of 300-900 $\mathrm{nm}$. The polymerization kinetics were monitored by changes in absorption of samples at $760 \mathrm{~nm}$.

\section{Fourier Transform Infrared Spectroscopy}

Fourier transform infrared spectroscopy (FTIR) spectroscopy was performed by the conventional procedure using $\mathrm{KBr}$ pellets on a Nicolet iS10 infrared spectrophotometer (Thermo Nicolet, USA). 


\section{SEM}

The surface morphologies of the cotton samples were visualized using an FEI Quanta-200 scanning electron microscope (FEI Company, the Netherlands).

Cyclic Voltammetry Tests

Cyclic voltammetry tests were performed using a CHI 660D electrochemical workstation $(\mathrm{CH}$ Instruments, Inc., Austin, USA) at room temperature to confirm the electroactive nature of the PANI/SDBS complex and the cotton samples. Before the measurement of the electrochemical activity of the PANI/SDBS complex, the unreacted monomers and oligomers that had formed were removed from the sample to prevent its electrochemical polymerization. The PANI/ SDBS complex was precipitated with acetone, followed by washing and drying, as described above. After that, it was redissolved in dimethyl sulfoxide, applied to the glassy-carbon electrode, and dried again to obtain a film. Before the measurement of the electrochemical activity of the cotton samples, a small piece of cotton sample was fixed to the glassy-carbon electrode surface by drying Nafion emulsion (1.5 wt\%), which possesses good cationexchange properties, biocompatibility, and film-forming properties and has been widely applied in the fields of fuel cells and sensors [18].

\section{Electrochemical Characterization of Conductive Cotton}

The antistatic properties of cotton samples were studied using a YG(B)342D Fabric Inductance-Type Electrostatic Tester (Wenzhou Darong Textile Instrument Co., Ltd., China), and their resistances were measured using a ZC-90G High Insulation Resistance Measuring Instrument (Yangzhou Subo Electric Co., Ltd., China), with two probes put on the surface of the cotton sample at a distance of $1 \mathrm{~cm}$.

The electromagnetic shielding effects of cotton samples were measured by the far-field EMC shielding effectiveness test fixture (DN1015 type) at low-frequency areas ranging from 2 to $100 \mathrm{MHz}$ in accordance with the ASTM D4935-99 standard test method [19].

\section{Results and Discussion}

Effect of Temperature on Polymerization

UV-vis absorption spectra of laccase-catalyzed aniline polymerization at different temperatures are shown in Fig. 1. The absorption bands at 430 and ca. $750-800 \mathrm{~nm}$ indicate the formation of a polaron in the polyaniline structure [20], which makes the polyaniline conductive. The maximum absorption peak of the polaron moved to the long wavelength part of the spectrum with the decreased temperature of the reaction solution, which reveals both the increase in its degree of polymerization and the alignment of the polymer structure. As the temperature increased, the degree of conjugation and conductivity of the polyaniline declined, and a brown by-product increased at the same time, which decreased the yield of polyaniline. Conductive polyaniline was not generated when the temperature exceeded $30{ }^{\circ} \mathrm{C}$ (Fig. 1). This result was inconsistent with the optimal temperature of laccase $\left(50{ }^{\circ} \mathrm{C}\right)$.

The synthesis of polyaniline is an exothermic reaction, which causes an increase in the temperature of the reaction system when the ambient temperature is constant. According to the van't Hoff rule, the reaction rate increases by two to four times with every $10{ }^{\circ} \mathrm{C}$ increase in 


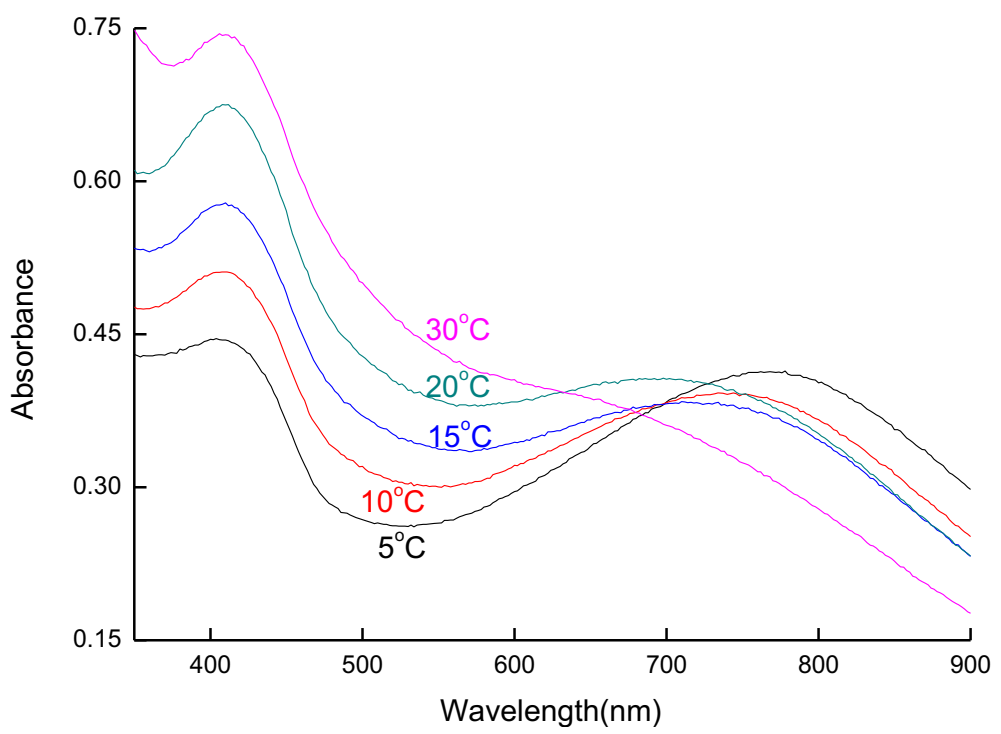

Fig. 1 UV-vis absorption spectra of laccase-catalyzed aniline polymerization at different temperatures. Experimental conditions: $50 \mathrm{mM} \mathrm{Na} \mathrm{HPO}_{4}$-citric acid buffered solution, $\mathrm{pH} 4.5$; [laccase] $=0.5 \mathrm{U} / \mathrm{mL}$; $[$ aniline $]=[\mathrm{SDBS}]=10 \mathrm{mM}$; reaction time $=8 \mathrm{~h}$. The solution was diluted with buffer $(1: 10)$

temperature. The interface could not reach a balanced structure, leading to the weakening of the head-to-tail coupling of monomers when polymerization was carried out at a high temperature. Tursun and Xiaogang [21] found that polyaniline prepared using a lowtemperature $\left(0{ }^{\circ} \mathrm{C}\right)$ solid-state polymerization process had a more uniform distribution of particles, greater crystallinity, and higher conductivity than polyaniline prepared using room temperature solid-state polymerization, because the increase in temperature led to a higher polymerization rate.

To improve the conductivity and the yield of polyaniline, the rate of aniline polymerization should be lowered and the monomer should be coupled head to tail in discontinuous form, according to the concepts of crystallography and the characteristics of the polymerization of aniline. For example, the polaron absorption maximum slowly shifted to the long wavelength part of the spectrum along with the increased polymerization time at $10{ }^{\circ} \mathrm{C}$ (Fig. 2), and the intensity of the polaron absorption maximum increased at the same time, which proved that the enzymatic synthesis of electroconductive polyaniline was slow and controllable.

It should be emphasized that the polyaniline in a complete oxidation state and the polyaniline in a complete deoxidation state may polymerize each other due to their different potentials, once the polymerization rate increases. This reaction leads to the head-to-head and tail-to-tail coupling of the monomers, reducing the conductivity of the polymer.

\section{Effect of $\mathrm{pH}$ on Polymerization}

To evaluate the effects of $\mathrm{pH}$ on the reaction course and the properties of the products formed, the laccase-catalyzed polymerization of aniline was carried out at different $\mathrm{pH}$ levels at $5{ }^{\circ} \mathrm{C}$ (Fig. 3). It is important to emphasize that the reaction is invertible as the $\mathrm{pH}$ varies, thus permitting a greater degree of control over the kinetics of the reaction [22]. At a $\mathrm{pH}$ of less than 4 , the solution was a milky turbid liquid and no product was generated. At a $\mathrm{pH}$ of more than 


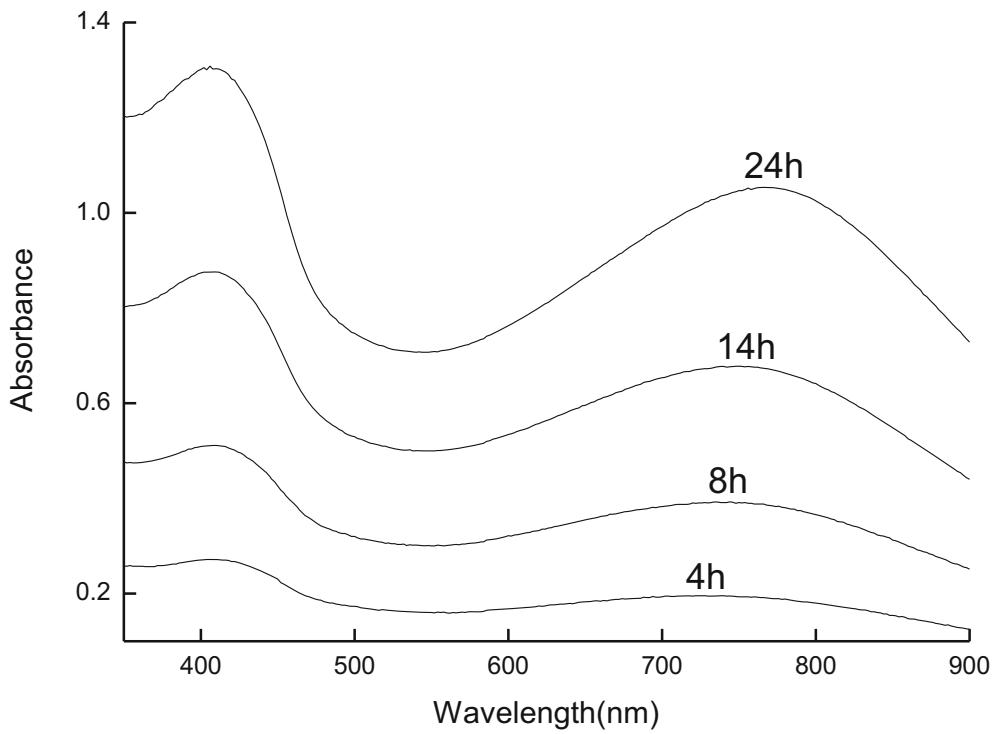

Fig. 2 UV-vis absorption spectra of laccase-catalyzed aniline polymerization at $10{ }^{\circ} \mathrm{C}$ with different reaction times

5, the absorption band at $800 \mathrm{~nm}$ disappeared and the brown by-product increased. The polymer had a high degree of conjugation and high conductivity when the polymerization was carried out at $\mathrm{pH}$ levels between 4.3 and 4.5 , which indicated that laccase from Aspergillus catalyzed the polymerization of aniline in the presence of sodium dodecylbenzenesulfonate micellar solution to form water-soluble conductive

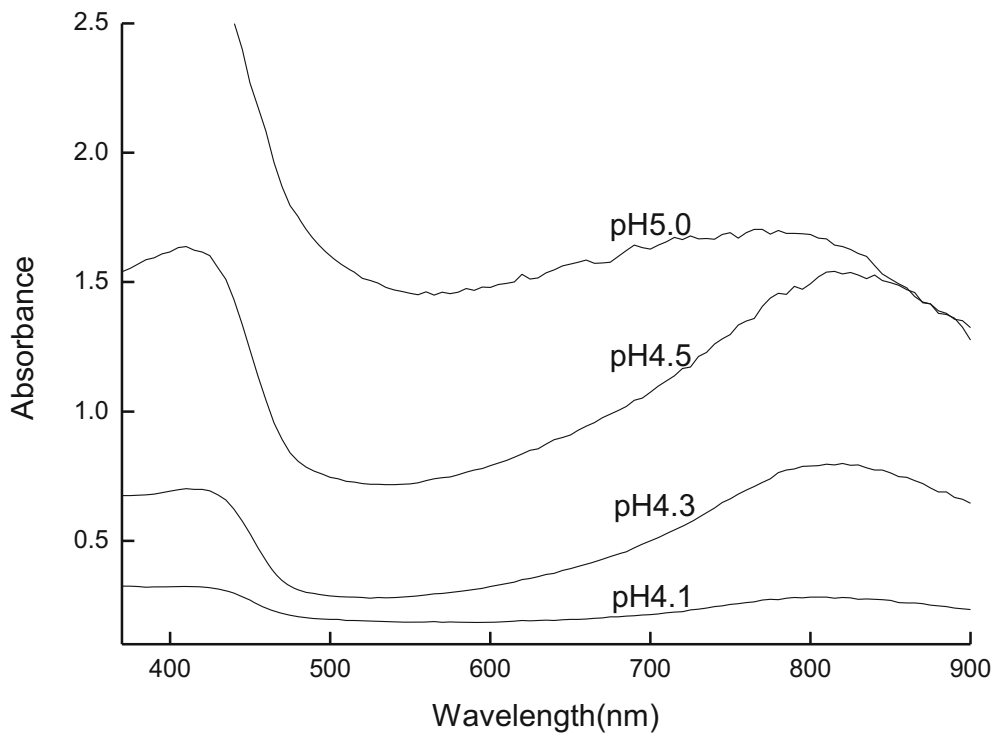

Fig. $3 \mathrm{UV}$-vis absorption spectra of laccase-catalyzed aniline polymerization at different $\mathrm{pH}$ levels. Experimental conditions: $50 \mathrm{mM} \mathrm{Na} 2 \mathrm{HPO}_{4}$-citric acid buffered solution; [laccase] $=0.5 \mathrm{U} / \mathrm{mL}$; [aniline] $=[\mathrm{SDBS}]=$ $10 \mathrm{mM}$; reaction time $=72 \mathrm{~h}$. The product was diluted with buffer $(1: 10)$ 


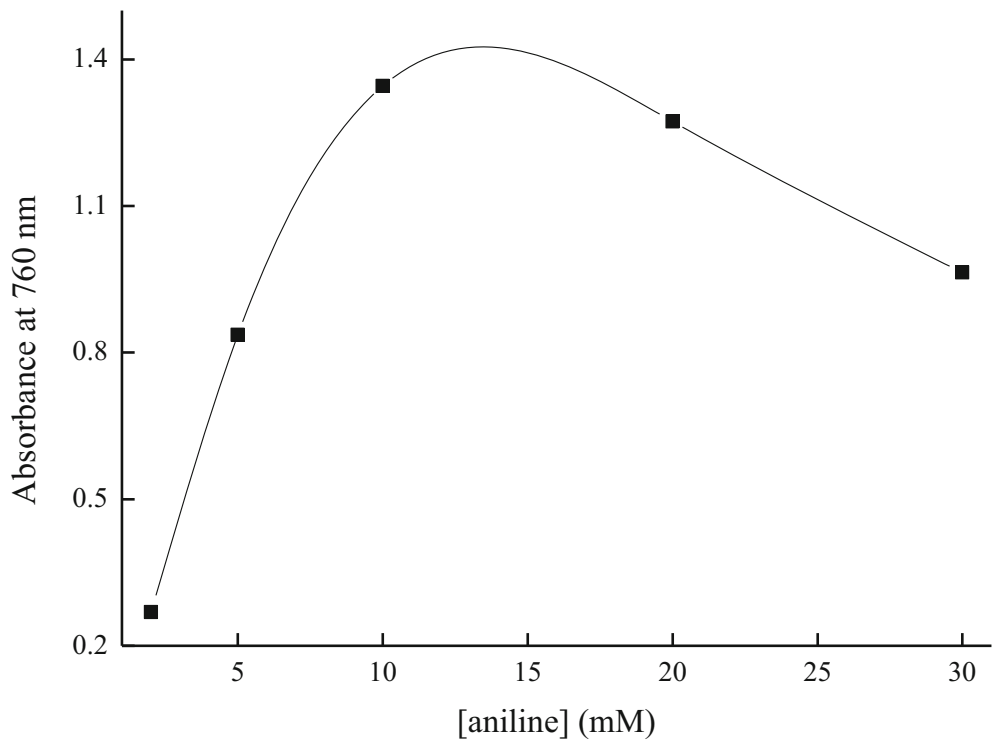

Fig. 4 Effect of concentration of aniline on the rate of aniline polymerization. Experimental conditions: $50 \mathrm{mM}$ $\mathrm{Na}_{2} \mathrm{HPO}_{4}-$ citric acid buffered solution; [laccase $]=0.5 \mathrm{U} / \mathrm{mL} ;[\mathrm{SDBS}]=10 \mathrm{mM}$; reaction time $=72 \mathrm{~h}$. The product was diluted with buffer $(1: 10)$

polyaniline under milder conditions than those of traditional chemical and enzymatic catalysis methods that have been reported $[11,13]$. Simultaneously, laccase had a high level of enzyme activity under these conditions.

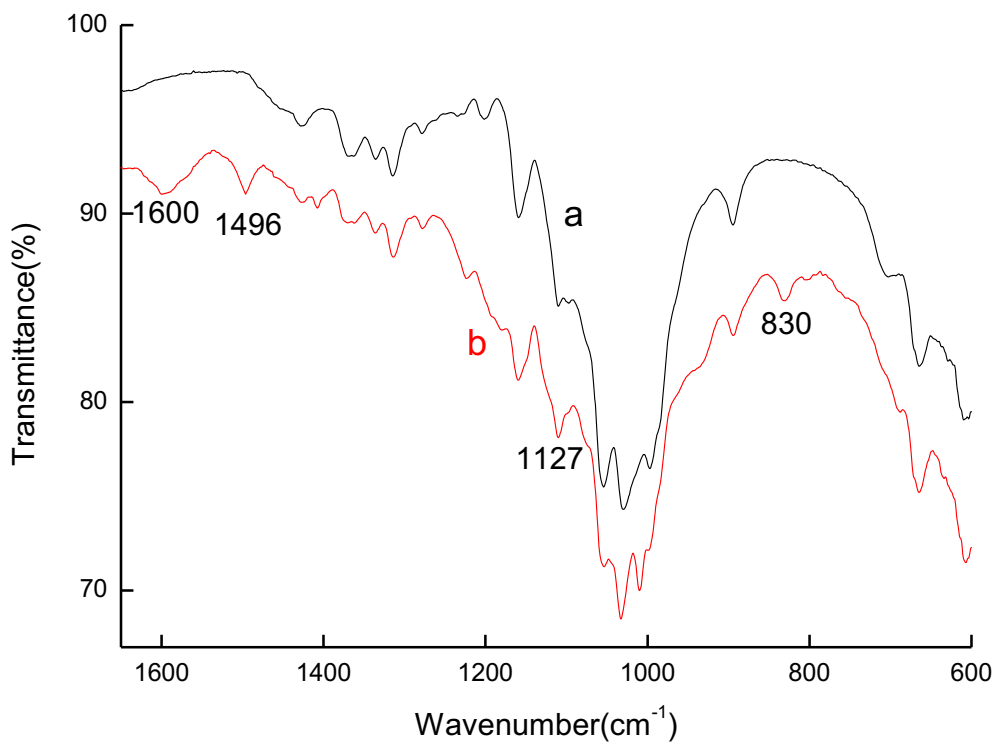

Fig. 5 FTIR spectra of the untreated cotton sample $(a)$ and conductive cotton $(b)$ 

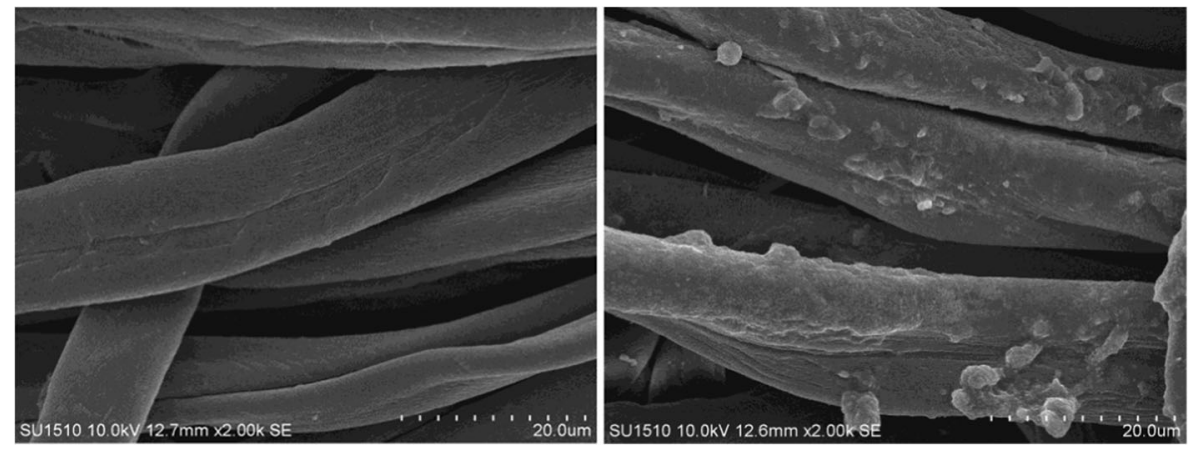

Fig. 6 SEM images of the untreated cotton sample (a) and conductive cotton (b)

Effect of Aniline and SDBS Concentrations and Ratio on Polymerization

The ratio of aniline to SDBS and the concentrations of aniline and SDBS were optimized according to the experiments described in Streltsov et al.'s work [11]. The synthesis was first carried out via varying the concentrations of aniline and SDBS from 3 to $20 \mathrm{mM}$ with a constant molar ratio of 1:1 (date not shown) and then conducted via varying the aniline concentration from 2 to $30 \mathrm{mM}$ with a constant SDBS concentration (Fig. 4). Although an increase in aniline content relative to SDBS enhanced the formation of longer linear polyaniline chains, a large excess of aniline would neutralize the negative charge of micelles, which led to the precipitation of complexes. Therefore, the concentration of aniline optimal for the synthesis is $10 \mathrm{mM}$.

\section{Structure and Morphology of Conductive Cotton}

The FTIR spectra of conductive cotton and a reference sample are shown in Fig. 5. The peaks at 1,600 and $1,496 \mathrm{~cm}^{-1}$ are attributable to the stretching of a $\mathrm{C}=\mathrm{C}$ ring in the quinoid and benzenoid units of the polyaniline [23]. The peak at $1,127 \mathrm{~cm}^{-1}$ is attributable to the $\mathrm{S}=\mathrm{O}$ stretching mode of sulfonic acid, which indicated the presence of SDBS in the complex and confirmed that the polyaniline was in doped form [24]. The head-to-tail coupling of the monomers, which led to the formation of linear polymeric chains, is indicated by the peak at $830 \mathrm{~cm}^{-1}$ [25]. These peaks confirmed the existence of conductive polyaniline on the surface of the conductive cotton. No polyaniline bands were visible in the spectrum of the reference sample.

As shown in Fig. 6, the morphology of conductive cotton was investigated using SEM imagery. After in situ polymerization of aniline, many granules of SDBS-doped PANI formed on the cotton surface, which enhanced the electrical properties of cotton. The hydrogen bonds

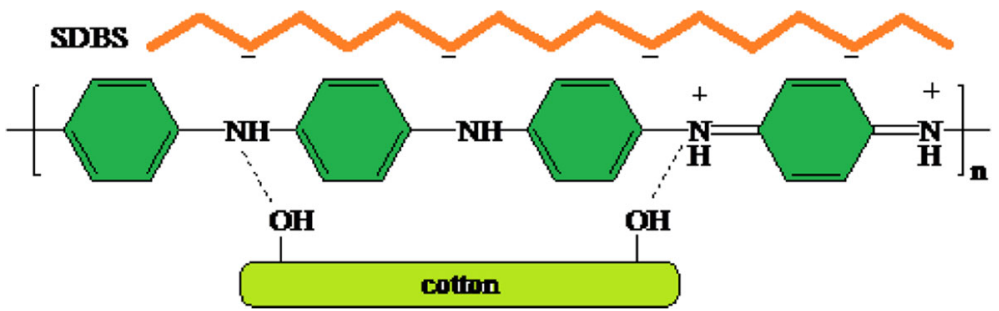

Fig. 7 Structure of conductive cotton 


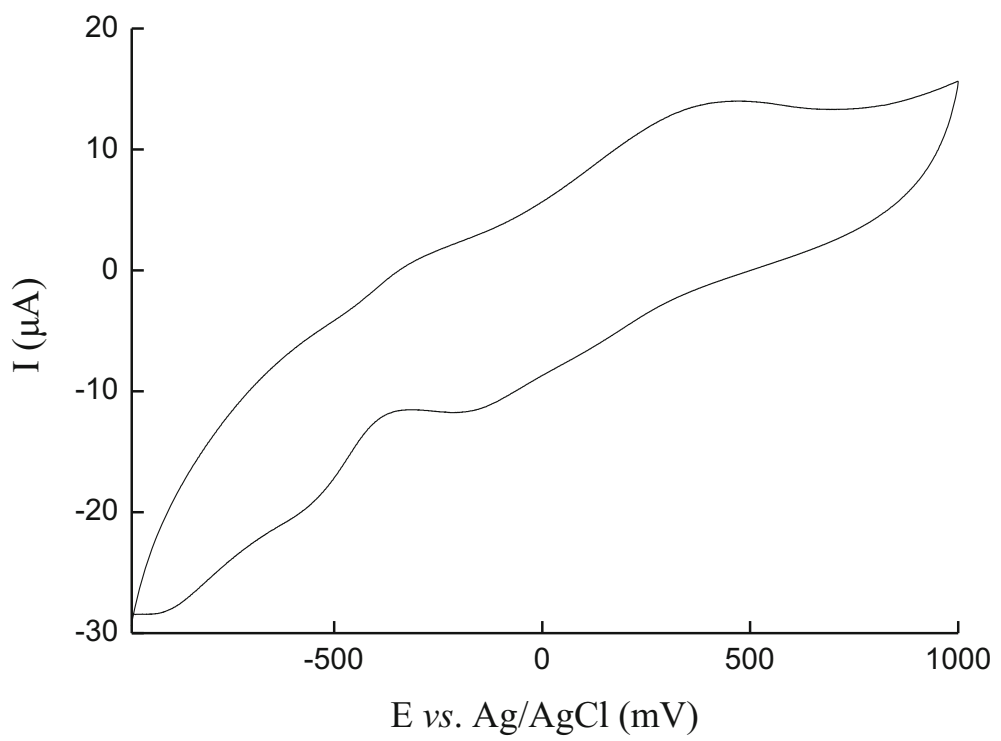

Fig. 8 Cyclic voltammograms of polyaniline films applied to the glassy-carbon electrode recorded at $\mathrm{pH} 4.5$

between the PANI and the hydroxyl groups were easy to form. In addition, the protonation of the PANI imine nitrogens through interactions with the hydroxyl groups of the cotton fibers strengthened the interactions between the PANI chains and the cotton. The interactions were also a result of lignosulfonate-doped polyaniline between the PANI chains and the hydroxyl groups present in lignosulfonate [26]. The structure is suggested in Fig. 7.

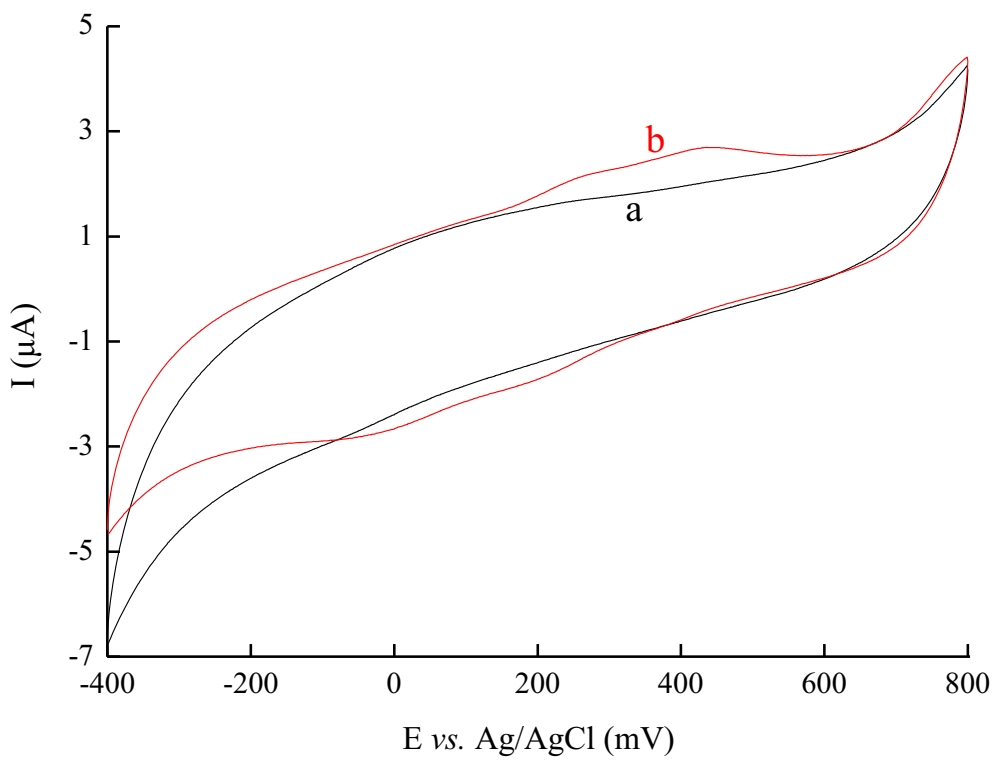

Fig. 9 Cyclic voltammograms of the untreated cotton sample $(a)$ and conductive cotton $(b)$ recorded at $\mathrm{pH} 4.5$ 
Table 1 Antistatic properties of cotton samples

\begin{tabular}{llll}
\hline Parameter & \multicolumn{2}{l}{ Samples } & \\
\cline { 2 - 4 } & Cotton & Cotton-SDBS & Cotton-PANI/SDBS \\
\hline$U, \mathrm{~V}$ & 2,652 & 2,548 & 725 \\
$t_{1 / 2}, \mathrm{~s}$ & 87 & 82 & 1.02 \\
\hline
\end{tabular}

Electrochemical Characterization of PANI/SDBS Complexes and Conductive Cotton

The SDBS-polyaniline complex in $50 \mathrm{mM} \mathrm{Na}_{2} \mathrm{HPO}_{4}$-citric acid buffered solution ( $\mathrm{pH} 4.5$ ) was electrochemically active, as can be seen from its cyclic voltammogram in Fig. 8. An oxidation peak and a reduction peak were observed at potentials of about 480 and $-150 \mathrm{mV}$, respectively. This pair of reversible symmetrical redox peaks confirmed that the SDBS-doped PANI possessed a high degree of electrochemical activity, which indicates the potential for using this type of polymer in electronic devices [24]. The conductive cotton in $50 \mathrm{mM}$ $\mathrm{Na}_{2} \mathrm{HPO}_{4}$-citric acid buffered solution ( $\mathrm{pH} 4.5$ ) was also electrochemically active, as can be seen from Fig. 9. An oxidation peak and a reduction peak were observed at potentials of ca. 470 and $-80 \mathrm{mV}$, respectively. This pair of reversible symmetrical redox peaks confirmed the electrochemical activity of the conductive cotton, illustrating its potential application in flexible electronic devices.

After in situ polymerization of aniline, the SDBS-doped PANI was immobilized on the cotton surface and the resistance of cotton samples decreased from $1.0 \times 10^{13}$ to $1.0 \times 10^{7} \Omega$. Further, the antistatic properties of the cotton samples are shown in Table 1. The values for peak voltage $(U)$ and half-life periods of charges $\left(t_{1 / 2}\right)$ on the fabrics' surfaces were significantly lower for conductive cotton compared with the other samples. Therefore, conductive

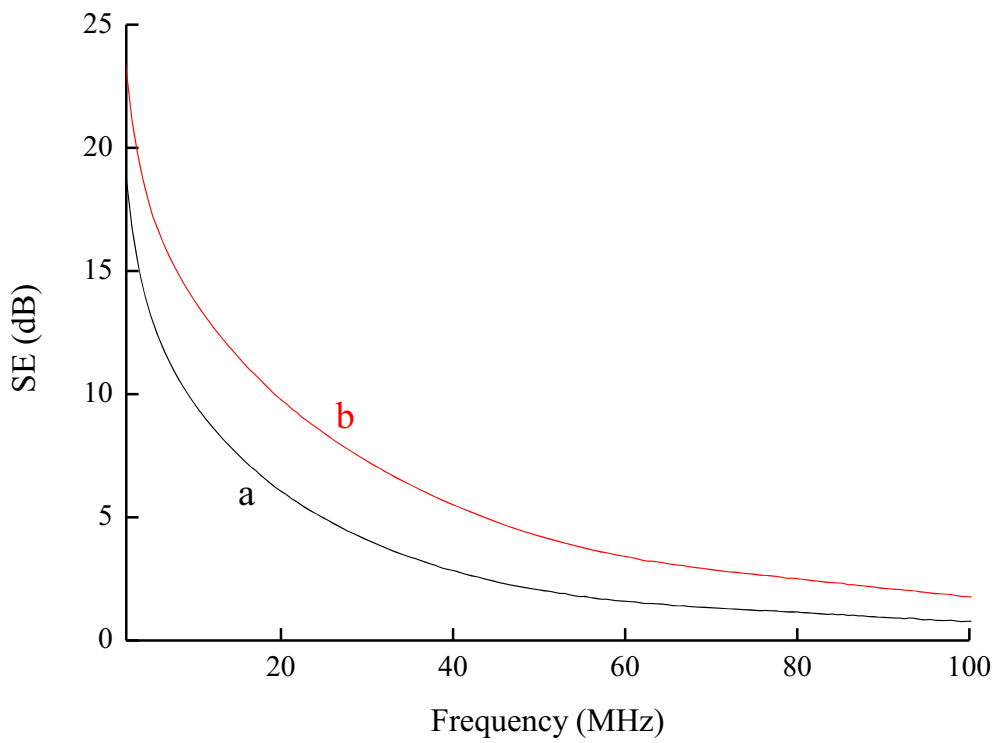

Fig. 10 Electromagnetic shielding of the untreated cotton sample $(a)$ and conductive cotton $(b)$ 
cotton prepared by polyaniline in situ polymerization using laccase as a catalyst had good antistatic performance.

The electromagnetic shielding effects (SE) of cotton samples are shown in Fig. 10. It could be seen that the shielding effect of cotton samples decreased with a frequency that ranged from 2 to $100 \mathrm{MHz}$ and the $\mathrm{SE}$ value of conductive cotton was much higher than that of the untreated cotton sample. In particular, the $\mathrm{SE}$ value of conductive cotton was about $4 \mathrm{~dB}$ higher than that of the untreated cotton sample at $20 \mathrm{MHz}$. These figures indicated the conclusion that the electromagnetic shielding effect of the cotton sample was enhanced after polyaniline in situ polymerization using laccase as a catalyst.

\section{Conclusion}

For this study, conductive cotton was prepared by polyaniline in situ polymerization, based on laccase-catalyzed synthesis of conducting PANI. This method had remarkable advantages as compared with both chemical and peroxidase-catalyzed methods due to the use of atmospheric oxygen as the oxidant. It was found that laccase from Aspergillus catalyzed the polymerization of aniline to form water-soluble conductive polyaniline under milder conditions than those of other methods which have been reported. The SDBS-doped PANI had good conductivity and a high yield at $\mathrm{pH} 4.5$ and a temperature of $5{ }^{\circ} \mathrm{C}$. Cyclic voltammetry indicated that the SDBSdoped PANI exhibited high electrochemical activity and may be used in electronic devices. Further, the SDBS-doped PANI was immobilized on the cotton surface after in situ polymerization of aniline, which significantly enhanced the electrical properties of the cotton, such as the antistatic property and the electromagnetic shielding effect. The research illustrates the potential for using common textile materials in flexible electronic devices.

Acknowledgments This work was financially supported by the National Natural Science Foundation of China (21274055, 51173071), the Program for New Century Excellent Talents in University (NCET-12-0883), the Natural Science Foundation of Jiangsu Province (BK2011157), the Fundamental Research Funds for the Central Universities (JUSRP51312B), and the Program for Changjiang Scholars and Innovative Research Team in University (IRT1135).

\section{References}

1. Anand, J., Palaniappan, S., \& Sathyanazayana, D. N. (1998). Progress in Polymer Science, 23, 993-1018.

2. Soto-Oviedo, M. A., Araujo, O. A., Faez, R., et al. (2006). Synthetic Metals, 156, 1249-1255.

3. Ahmad, N., \& MacDiarmid, A. G. (1996). Synthetic Metals, 78, 103-110.

4. Karami, H., Mousani, M. F., \& Shamsipur, M. (2003). Journal of Power Sources, 117, 255-259.

5. Parente, A. H., Marques, E. T. A., Azevedo, W. M., et al. (1992). Applied Biochemistry and Biotechnology, 37, 267-273.

6. Kaneto, K., \& Kaneko, M. (2001). Applied Biochemistry and Biotechnology, 96, 13-23.

7. Venancio, E. C., Pencheng, W., \& MacDiarmid, A. G. (2006). Synthetic Metals, 156, 357-369.

8. Qingfu, X., Haijun, Z., et al. (2012). Modern Chemical Industry, 32, 13-17.

9. Dan, C., Yue-E, M., \& Tianxi, L. (2013). ACS Applied Materials \& Interfaces, 5, 1206-1212.

10. Schnippering, M., Powell, H. V., Mackenzie, S. R., et al. (2009). Journal of Physical Chemistry C, 113, 20221-20227.

11. Streltsov, A. V., Shumakovich, G. P., Morozova, O. V., et al. (2007). Applied Biochemistry and Microbiology, 44, 264-270.

12. Desai, S. S., \& Nityanand, C. (2011). Asian Journal Biotechnology, 3, 98-124.

13. Longoria, A. M., Hailin, H., \& Vazquez-Duhalt, R. (2010). Applied Biochemistry and Biotechnology, 162, 927-934. 
14. Valderrama, B., Marcela, A., \& VazquezDuhalt, R. (2002). Chemistry and Biology, 9, 555-565.

15. Chaubey, A., Pande, K. K., Pandey, M. K., et al. (2001). Applied Biochemistry and Biotechnology, 96, 239-248.

16. Otrokhov, G. V., Morozova, O. V., Vasil'eva, I. S., et al. (2013). Biochemistry (Moscow), 78, 1539-1553.

17. Xuejiao, S., Rubing, B., Ya, Z., et al. (2013). Applied Biochemistry and Biotechnology, 171, 1673-1680.

18. Juan, W., Hongbin, Y., Dian, H., et al. (2012). Journal of the American Chemical Society, 4, 1963-1971.

19. Richeng, Y., Zhenghou, Z., \& Hui, S. (2011). Advances in Materials Research, 306-307, 848-851.

20. Karamyshev, A. V., Shleev, S. V., Koroleva, O. V., et al. (2003). Enzyme and Microbial Technology, 33, 556-564.

21. Tursun, A., \& Xiaogang, Z. (2004). Journal of Functional Materials, 35, 330-332.

22. Wei, L., Kumar, J., Tripathy, S., et al. (1999). Journal of the American Chemical Society, 121, 71-78.

23. Louarn, G., Lapkowski, M., Quillard, S., et al. (1996). Journal of Physical Chemistry C, 100, 6998-7006.

24. Xingui, L., Meirong, H., Jianfeng, Z., et al. (2004). Colloids and Surfaces, A: Physicochemical and Engineering Aspects, 248, 111-120.

25. Lim, C. H., \& Yoo, Y. J. (2000). Process Biochemistry, 36, 233-241.

26. Shao, L., Qiua, J. H., Feng, H. X., et al. (2009). Synthetic Metals, 159, 1761-1766. 